\title{
Understanding the Mechanisms Generating Outcomes in a Danish Peer support Intervention for Socially Vulnerable People with type 2-Diabetes: A Realist Evaluation
}

\section{Stine Dandanell Garn ( $\nabla$ soga@sund.ku.dk)}

University of Copenhagen Department of Public Health: Kobenhavns Universitet Institut for Folkesundhedsvidenskab https://orcid.org/0000-0003-3503-9477

\section{Charlotte Glümer}

Aalborg University Department of Health Science and Technology: Aalborg Universitet Institut for Medicin og Sundhedsteknologi

\section{Sarah Fredsted Villadsen}

University of Copenhagen Department of Public Health: Kobenhavns Universitet Institut for

Folkesundhedsvidenskab

\section{Gritt Marie Hviid Malling}

University of Copenhagen Department of Public Health: Kobenhavns Universitet Institut for

Folkesundhedsvidenskab

\section{Ulla Christensen}

University of Copenhagen Department of Public Health: Kobenhavns Universitet Institut for Folkesundhedsvidenskab

\section{Research}

Keywords: Realist evaluation, Implementation, Mechanisms, Context, Peer support, Type 2-Diabetes Mellitus, Diabetes self-management, Healthcare services, Inequality, Complex interventions

Posted Date: February 26th, 2021

DOl: https://doi.org/10.21203/rs.3.rs-256521/v1

License: (1) This work is licensed under a Creative Commons Attribution 4.0 International License. Read Full License 


\section{Abstract}

Background: Despite increasing use and positive effects of peer support interventions, little is known about how they produce outcomes. Thus, it is essential not only to measure outcomes, but also to identify the mechanisms by which they are generated. Using a realist evaluation approach, we aimed to identify the mechanisms generating outcomes in a Danish peer support intervention for socially vulnerable people with type 2-diabetes (peers). By investigating how the peers interacted in the intervention, we further examined how peers' individual contextual factors facilitated or hindered the mechanisms in operation.

Methods: We used a multi-method case-study design $(n=9)$. Data included semi-structured interviews with four key groups of informants (peer, peer supporter, project manager and a diabetes nurse) for each case $(n=25)$. Furthermore, we collected survey data from peers both before and after participation $(n=9)$. The interview data were analysed using a systematic text condensation, and the Intervention-context-actormechanism-outcome framework was used to structure the analysis.

Results: We identified two groups of mechanisms that improved diabetes self-management and the use of healthcare services (outcomes): 'perceived needs and readiness' and 'encouragement and energy'. However, the mechanisms only generated the intended outcomes among peers with a stable occupation and financial situation, a relatively good health condition, and sufficient energy (all defined as contextual factors). Independent of these contextual factors, 'experience of social and emotional support' was identified as a mechanism within all peers that increased self-care awareness (defined as output). Dependent on whether the contextual factors facilitated or hindered the mechanisms to generate outcomes, we categorised the peers into those who achieved outcomes and those who did not.

Conclusions: We identified two groups of mechanisms that improved the peers' diabetes selfmanagement and use of healthcare services. The mechanisms only generated the intended outcomes if peers' individual contextual factors facilitated an active interaction with the elements of the intervention. However, independent of these contextual factors, a third group of mechanisms increased self-care awareness among all peers. We highlight the importance of contextual awareness of the target groups in the design and evaluation of peer support interventions for socially vulnerable people with type 2diabetes.

Trial registration: ClinicalTrials.gov, Retrospective Registration (01/20/2021), registration number NCT04722289, https://clinicaltrials.gov/ct2/show/NCT04722289?

term=The+Together+on+Diabetes+Intervention+-+a+Realist+Evaluation\%2C\&draw=2\&rank=1

\section{Contribution To The Literature}

- Contextual awareness is key to the successful implementation of complex health interventions. The influence of contextual factors on the outcome-generating mechanisms is poorly explored, especially in relation to the target groups. 
- Using a realist evaluation approach, including the Intervention-context-actor-mechanism-outcome framework (ICAMO) framework, we illustrate how mechanisms in a peer support intervention for socially vulnerable type 2-diabetes patients only generated intended outcomes if peers' contextual factors facilitated their interactions in the intervention.

- Within the field of implementation science, we contribute with novel, in-depth knowledge on the importance of contextual awareness of the target groups in designing and evaluating complex health interventions.

\section{Background}

Peer support programmes are increasingly used worldwide as a supplement to the established healthcare system to support people with type 2-diabetes (T2D) in managing their disease (1-4). Moreover, these are acknowledged by the World Health Organization as feasible and cost-effective interventions (5). Peer support within the healthcare field refers to social, emotional and practical assistance provided by nonprofessionals to help people adopt, change and maintain health behaviours $(6,7)$.

Several studies have found a positive effect of peer support programmes on diabetes self-management (DSM), such as meal planning, physical activity, taking medication and blood glucose monitoring (8-11). Furthermore, peer support has benefitted health outcomes, such as self-reported health status, emotional distress and glycaemic and blood pressure control $(8,9,11-13)$. Finally, peer support is highlighted for its potential to support socially vulnerable people in managing their T2D (14-16). Often, this group is referred to as people with low socioeconomic status, such as low levels of income, education, employment and social relations $(14,16-18)$. This population group have a higher risk of developing complications of T2D $(15,19)$, often face multiple barriers to accessing healthcare services $(14,15,20)$ and experience worse health outcomes (21). However, even though socioeconomic differences exist in access to healthcare services, as well as the treatment and consequences of T2D $(16,19,20,22)$, no studies have, to our knowledge, focused on the implementation of peer support programmes for socially vulnerable people with T2D.

In general, little is known about how peer support interventions produce outcomes. Methodological guidance on complex interventions emphasises the importance of focusing on what is implemented, including the mechanisms that generate outcomes and the contexts that influence their implementation $(23,24)$. Furthermore, as described in The Standards for Reporting Implementation Studies (StaRI) (25), to understand and interpret effect, it is essential not only to measure the outcome but also to underpin the mechanisms that generate it and investigate the influence of the implementation context. Using the realist evaluation (RE) approach developed by Pawson \& Tilley (26), it is possible to identify outcomegenerating mechanisms in complex health interventions such as peer support programmes. This can be achieved by investigating how the actors involved interact in the intervention and how contextual factors influence this. The RE's main principle is that all complex interventions have underlying theories, referred to as programme theories that describe the relationship between context, mechanisms and outcomes (CMO-configurations) in the intervention. By developing and empirically testing a programme theory, it is 
possible to understand what works, how, under which conditions and for whom (26). Also, the "Intervention-Context-Actor-Mechanism-Outcome" (ICAMO) framework (27) is increasingly applied as a supplement (27-29). The ICAMO is a modified version of Pawson \& Tilley's CMO-configuration, as it includes two extra components into the configuration: "intervention" and "actors". Using the ICAMO, it gives an explicit focus on how the actors involved interact in the intervention.

Study aim

By using the RE approach, including the ICAMO-framework, we aimed to identify the mechanisms generating outcomes in a Danish peer support intervention aiming to improve diabetes self-management and the use of healthcare services among socially vulnerable people with type 2-diabetes (peers). By investigating how the peers interacted in the intervention, we examined how their individual contextual factors facilitated or hindered the operation of mechanisms in the intervention.

\section{Methods}

Setting: the Danish healthcare system

The Danish healthcare system is universal and almost free of charge for Danish citizens, as all healthcare services are financed by general taxes (30). It operates across three political and administrative levels: the state, five regions and 98 municipalities. The state is responsible for initiating and coordinating overall national health policies and legislation on healthcare. The regions are responsible for the planning and execution of diabetes care within hospitals and general practice, and the municipalities are responsible for diabetes rehabilitation outside hospitals, health promotion and disease prevention $(31,32)$.

'Together on Diabetes' intervention

Together on Diabetes is an ongoing intervention, developed and implemented in 2017 by Copenhagen Municipality's Centre for Diabetes (CFD) and The Danish Diabetes Association (DDA). The intervention is part of the Copenhagen city action plan for T2D (32), and, the public-private partnership 'Cities Changing Diabetes Copenhagen' (CCDC) $(33,34)$.

Through social, emotional and practical support provided by non-professional volunteers with T2D ("peer supporters"), the intervention aims to improve DSM and increase the use of healthcare services among socially vulnerable people with T2D (peers).

The intervention consists of five components: recruitment of peers and peer supporters; training of peer supporters; matchmaking between peers and peer supporters; 6 months of fortnightly individual face-toface meetings between peers and peer supporters; and ongoing supervision and network meetings for peer supporters. The peer support meeting contains three activities: 'social and emotional support'; 'assistance in daily management'; and 'linkage to healthcare services' (35). 
Based on an initial quantitative analysis of people with T2D in disadvantaged areas of Copenhagen with high diabetes prevalence (22), the inclusion criteria to participate as a peer were defined as poorly regulated T2D, multi-morbidity, no employment, low/no education, no contact to the healthcare system and living alone with no/minimal social network. The exclusion criterion was poorly regulated mental disease.

The inclusion criteria to become a peer supporter were defined as well-regulated T2D, basic knowledge about T2D and the Danish healthcare system, good communication skills, empathy and an interest in supporting a socially vulnerable person with T2D. These criteria were based on existing knowledge about peer support programmes $(6,36,37)$.

As part of the intervention's development and implementation, CFD and DDA formulated an initial programme theory that included the intervention components and theoretical assumptions of intended outcomes. Further, potential contextual factors that could affect their implementation. These included both organisational and interpersonal contextual factors, such as resources, coordination and communication with internal and external stakeholders, timing of the intervention, and the relationship and chemistry between peer and peer supporter. Furthermore, individual contextual factors are included, such as the peers and peer supporters' sociodemographic characteristics, health condition, other life events and social relations. The initial programme theory did not include mechanisms; hence, the aim of this study was to identify and investigate mechanisms.

Conceptual framework

Based on the initial programme theory and relevant literature within the field $(6,14,16,36,37)$, we developed an initial ICAMO model for this study (Fig. 1) to structure our theoretical assumptions of how the intervention worked, for whom and under what conditions. The initial ICAMO model focused on a specific part of the intervention: the relationship between the three intervention activities in the peer support meetings, the mechanisms within peers that generated outcomes and the individual contextual factors in peers' everyday lives that influenced how the mechanisms were at stake.

As illustrated in Fig. 1, we used Pawson's definition of intermediate outputs (cognitive changes) and outcomes (behavioural changes) (38) to distinguish between whether the mechanisms generated both the intended cognitive and behavioural changes in terms of improved DSM and use of healthcare services, or if they solely generated cognitive changes. Moreover, we used Pawson's four layers of contextual factors by Pawson (38) (the individual, interpersonal, organization and infrastructure layers), focusing on the individual layer, to investigate how the peers' sociodemographic characteristics, capacities and life circumstances influenced how they interacted in the intervention. Finally, to define the term 'mechanism', we applied the mechanism-framework by Dalkin et al. (39). The framework is based on the original work of Pawson \& Tilley (26) and defines mechanism as "a combination of resources offered by the social programme under study and stakeholders' reasoning in response" (39). Following this framework, a mechanism is both the resource that an intervention provides and the recipients' reasoning and response to it. According to Dalkin et al. (39), this relationship between intervention resources that 
are introduced into a specific context enhances changes in the recipients' reasoning that create the mechanisms that cause the outcomes, thereby making the intervention work.

Study design, case selection and recruitment

We used a multi-method case study approach $(40,41)$ to identify the mechanisms that generated the intended outcomes. Furthermore, we used the ICAMO framework to structure our analysis.

All peers and peer supporters included and matched in the intervention from February 2018 to July 2019 were invited by the project manager to participate in this study. In total, 9 out of 12 pairs completed and accepted the invitation and were consequently selected as cases. The majority of peers were recruited through CFD $(\mathrm{N}=7)$. They were recruited among people who were considered too vulnerable to participate in CFD's regular diabetes education and rehabilitation services. The remaining peers were recruited either by their general practitioner $(\mathrm{GP})(\mathrm{N}=1)$ or through home care $(\mathrm{N}=2)$.

The peer supporters were recruited among people who had previously participated in CFD's services ( $\mathrm{N}=$ 5) by members from the DDA $(N=2)$, the $G P(N=1)$, or by people who applied via the 'Together on Diabetes' webpage $(\mathrm{N}=2)$.

Data collection

The study consisted of a quantitative survey and qualitative semi-structured interviews. Both were conducted between February 2018 and April 2020.

\section{Surveys}

A quantitative survey was conducted among peers at baseline and follow-up $(N=9)$ to measure both improvements in their DSM and use of healthcare services (outcomes). Questions from the Danish National Health Survey (42) were used to measure DSM: (eating habits, physical activity, medication intake and blood sugar monitoring). Furthermore, the use of healthcare services was measured by the number of times (during a 12-month period) the peers' attended diabetes-related appointments with GPs, food therapists and ophthalmologists, or had other form of contact with relevant healthcare services. Also, survey data were used to obtain information on peers' individual contextual factors, such as their sociodemographic characteristics, co-morbidity, diabetes complications, social relation, and other life events. A diabetes nurse from CFD conducted the survey as a structured interview, as the peers were considered too vulnerable to complete the survey on their own. The diabetes nurse visited the peers in their own homes, both before and after the intervention. The peers received a handout version of the survey, so they also were able to read the questions themselves.

Individual semi-structured interviews

We conducted 25 individual semi-structured interviews across the nine cases. The informants consisted of the peers $(n=9)$, peer supporters $(n=10)$, project manager and diabetes nurse. We interviewed each 
type of informant per case to obtain different perspectives on how the peers' interacted in and benefited from the intervention. The interviews were conducted both immediately after the 6-month intervention and after the follow-up survey was completed.

The interview guide was semi-structured and based on survey data and the initial programme theory. The interviews consisted of questions about the following topics: peers and peer supporters' reasons for participation; how they had been recruited; peers' perceived needs towards the intervention; experiences of and activities conducted in the meetings; how they experienced peers' cognitive (output) and behavioural changes (outcomes) as a result of the intervention; and whether any barriers or facilitators in peers' contexts had affected their interaction in the intervention. In addition, the interviews with the project manager and diabetes nurse included questions about their reflections on patterns in outputs, outcomes and contextual factors across the nine cases. During the interviews, only the informant and interviewer were present. Interviews with peers took place in their own homes. Interviews with the other informants took place at CFD. However, due to the COVID-19 pandemic, seven interviews (peers $(n=3)$, peer supporters $(n=3)$ and project manager $(n=1))$ were collected by telephone.

\section{Ethical Considerations}

The present study is part of a larger evaluation study of three diabetes interventions developed within the CCDC partnership programme (34). The study was approved by the Danish Data Protection Agency (Rec. No: 2015-55-0630) and followed the codes of ethics in the Helsinki II Declaration.

An ethical application was sent to The Research Ethics Committee for SCIENCE and HEALTH, at the Capital Region of Denmark.

The Research Ethics Committee reported that they did not identify any ethical hindrances in conducting the study (Id. No: 18029206) and decided that no formal ethical approval was needed.

All participants received written and verbal information about the study and gave written consent to participate.

They were guaranteed anonymity and informed that they could withdraw from the study at any time, should they wish to do so.

\section{Data analysis}

The semi-structured interviews were audio-recorded and transcribed verbatim. All transcripts were managed in NVivo 12 (43). The Systematic Text Condensation (44) was used to analyse data and the ICAMO framework was used to structure the analytical findings (27).

The analysis consisted of four steps:

1. Reading all transcripts to obtain an overall impression of data and identify preliminary themes related to the study aim. 
2. Identifying meaning units related to the preliminary themes. The first and last author then developed a set of codes, which they compared and discussed to clarify the relationship between the intervention activities, the mechanisms within peers that generated outcomes and the contextual factors in peers' everyday lives that influenced how the mechanisms were at stake.

This ensured confidence in the findings and facilitated agreement on an initial coding framework.

3. Having coded all of the transcripts, we organised and synthesised data into themes with similar code groups. We then selected quotes to illustrate the findings.

4. We revised our initial ICAMO model based on our empirical findings.

Throughout our study design, data collection and analysis, we followed the reporting standards for realist evaluations developed by the RAMESES II (45) and the The Standard for Reporting Implementation Studies (STaRI)(25).

\section{Results}

Study participants

As illustrated in Table 1, the 9 peers included 7 males and 2 females. They were primarily male, middleaged, of Danish origin, outside of the labour market, and with short and intermediate education backgrounds as well as multiple diagnoses and diabetes complications. 
Table 1

Peers' sociodemographic characteristics

\begin{tabular}{|ll|}
\hline & Peers \\
\hline N & 9 \\
\hline Sex & 7 \\
Male & 2 \\
Female & \\
\hline Age & 1 \\
Below 50 & 6 \\
50-65 & 2 \\
Above 65 & \\
\hline Country of Birth: & 7 \\
Denmark & 1 \\
Other Western countries & 1 \\
Non-Western countries & \\
\hline Source of income & \\
Social security & 4 \\
State pension & 2 \\
Disability pension & 3 \\
\hline Education & 4 \\
Primary & 4 \\
Secondary & 1 \\
Higher & \\
\hline Living situation & \\
Alone & \\
With children & \\
With others & \\
\hline
\end{tabular}

(Baseline survey data) 


\begin{tabular}{|ll|}
\hline & Peers \\
\hline Other diagnoses $(\mathrm{N})$ & \\
Mental health disorders & 6 \\
Arthritis & 4 \\
Oral health problems & 3 \\
KOL & 2 \\
Other chronic diagnoses & 3 \\
\hline Diabetes complications $(\mathrm{N})$ & \\
Cardiovascular diseases & 7 \\
Hypertension & 5 \\
Neuropati & 4 \\
Nefropati & 4 \\
\hline (Baseline survey data) & \\
\hline
\end{tabular}

The ten peer supporters included 6 males and 4 females. They had different educational backgrounds, employment status and experience of working as a support volunteer. The diabetes nurse had 20 years of experience as a nurse and had previously worked as a home nurse. The project manager had 10 years of experience working with socially vulnerable groups and in peer support programmes.

Differences in peers' outcomes

Survey and interview data revealed large differences in peers' outcomes from the intervention. The intended outcomes (improved DSM and use of healthcare services) were only identified among four peers (See Figs. 2 and 3). A common pattern for those who achieved outcomes, compared to those who did not achieve any, was the implementation of a minimum of two of the three intervention activities in the peer support, meetings. In the four cases, the peer supporters had been providing social and emotional support assisted with daily tasks (grocery shopping, cleaning, cooking healthy meals and exercising) and/or acted as a link to healthcare services (that is, being an observer at GP appointments and assisting in communication with other relevant healthcare services). In contrast, in the five cases in which none of intended outcomes where achieved, the peer support meetings had mainly consisted of social and emotional support.

Contextual factors influencing variation in mechanisms and outcomes

Four main contextual factors in peers' everyday lives were found to explain why some peers achieved the intended outcomes and others did not: 'occupation and financial situation', 'health condition', 'energy' and 'other life events') (See Figs. 2 and 3). The peers who achieved the intended outcomes were characterised 
by a stable occupation and financial situation, receiving a state or disability pension, combined with being in a better state of health. Moreover, some peers' participation in other social activities during the intervention might have facilitated the outcomes. Conversely, for the peers, who did not achieve any of the intended outcomes, these contextual factors functioned as barriers to how the peers interacted in the intervention. This group was characterised by an unstable occupation and financial situation, receiving social security benefits, combined with being in a worse state of health with severe pain due to multiple diabetes complications and both psychical and chronic mental diagnoses. In addition, some experienced negative life events during the intervention, such as accidental falls and the death or illness of close relatives, which interrupted their participation. Due to these barriers, which were on the individual contextual level, many peers described how they lacked energy to interact in the intervention.

Mechanisms generating outputs and outcomes

Interview data revealed two groups of mechanisms within the peers that generated the intended outcomes: 'perceived needs and readiness' and 'encouragement and energy' (See Figs. 2 and 3). However, data showed a large variation in how these mechanisms operated depending on whether the contextual factors functioned as facilitators or barriers to the peers' interactions in the intervention. Independent of the influence from the contextual factors, a third mechanism, 'experience of social and emotional support', was identified within all peers that increased self-care awareness (output).

Perceived needs and readiness

Peers' perceived needs and readiness to interact in the intervention were found as interrelated mechanisms that generated both the intended cognitive (outputs) and behavioural changes (outcomes) in DSM and the use of healthcare services. However, as mentioned, these mechanisms operated differently between the peers depending on their context. For the peers where contextual factors in their everyday lives functioned as facilitators, a common pattern was that they had a perceived need for support in accessing and navigating the healthcare system and/or to improve central tasks in their DSM, for example, to get started with daily walks or cooking daily meals. In addition, they demonstrated a sufficient amount of readiness to interact in the intervention to meet these needs. This is illustrated in the following quote from one peer:

"I needed a little help to get started cooking for myself because it had come to a complete standstill (...) To get started with grocery shopping so I could start making some proper food."(Peer, outcomes achieved)

In contrast, the peers' perceived needs and readiness came into play more differently among those who were challenged by an unstable occupation and financial situation, a poor health condition, lack of energy and other negative life events. In general, they were less reflective about what they wanted to achieve with their participation. In the interviews, the majority expressed not having specific needs other than a need for social contact in their lives. Some mentioned not wanting to make health behaviour changes to improve their DSM or receive support in navigating the healthcare services. This is illustrated 
in the following case, where a peer explained how he did not want to stop drinking alcohol as he felt it did not matter anyway:

"I don't want to do that [stop drinking alcohol red.] (...) because I feel better when I drink. I am happy (...) Diabetes you have for life, no matter what you do, you know? So, so what? I am dying anyway." (Peer, no outcomes achieved)

According to the informants in this case, the peer's poor life circumstances were causes of this lack of need and readiness to stop drinking alcohol. When entering the intervention, he had severe mental and physical challenges due to several chronic diagnoses and diabetes complications. Moreover, he had recently lost his job and had to focus his sparse energy on attending meetings at the jobcentre. In the interview, he mentioned that he was worried about being evicted from his home if he could not pay the next month's rent. He had previously lived on the streets but was no longer capable of this due to his poor health condition. Thus, he was satisfied as long as he had a roof over his head.

"Nothing is clear, you know? (...) They can send me a letter tomorrow stating that I will no longer receive cash benefits." (Peer, no outcomes achieved)

Because of the peers' lack of need and readiness to achieve the intended outcomes, the peer support meetings mainly consisted of social and emotional support. This was a source of great frustration for many of the peer supporters, who felt unsuccessful in their roles while not being able to implement all three activities in the peer support meetings. One peer supporter put it this way:

"I was prepared for meeting someone who had a goal, who had signed up because he/she wanted to make improvements, and $X$ (the peer, red.), has never wanted that." (Peer supporter)

Encouragement and energy

Another group of interrelated mechanisms that generated both the intended cognitive and behavioural changes in DSM and use of healthcare services was the encouragement received from the peer supporter and the peer's level of energy to interact in the intervention. Encouragement from peer supporters to regularly attend diabetes-related appointments with the GP or get started with healthier eating or exercise patterns positively affected the peers' levels of energy to interact in the intervention and set meaningful, achievable goals. However, this was only seen in cases where peers' individual contextual factors facilitated this engagement. In the following interview excerpt, the diabetes nurse described a case where encouragement from the peer supporter to improve eating habits and attend diabetes-related appointments with the GP was the push needed for the peer to make these behavioural changes:

"Now, all of a sudden, there are some people around him who support him in the importance of visiting the GP (...). He knows what he has to do, but his challenge is in getting it done. (...) 'Together on Diabetes' has helped him get things done and that is what makes the big difference regarding his health, and health condition as I see it. It's simply a matter of getting that little push." (Diabetes nurse) 
In contrast, encouragement from the peer supporters did not generate outcomes among peers for whom contextual factors functioned as barriers. In the interviews, many described how their unstable financial situation, poor state of health condition, lack of energy and other life events resulted in them not being able to respond to their peer supporters' encouragement. As one peer described:

"I appreciate when somebody tries to help me, you know? Try to lift me (...) what you tell me now, I won't do tomorrow but it is on my mind, and I try (...)" (Peer, no outcomes achieved)

As this quote illustrates, the peer supporter's encouragement activates a reflection within the peer. However, the peer's challenging life circumstances and lack of energy constitute a barrier to respond and make the behavioural changes needed.

Experience of social and emotional support

The experience of receiving social and emotional support was found as a mechanism that generated increased self-care awareness (output). Unlike the other mechanisms, it was found within all peers regardless of their occupation and financial situation, health condition, amount of energy, and other life events. In the interviews, the peers emphasised how they valued the regular meetings with their peer supporter. In the majority of cases, these meetings were the only social contact they had. Many mentioned the importance of the peer supporter as a voluntary like-minded person that they could talk to about issues related to everyday life with T2D. One peer elaborated:

"When I talk with a person, who is like me, who has diabetes, it is easier to explain because he understands (...) because he has the same problems as me." (Peer, no outcomes achieved)

Several described being in the same situation as their peer supporter - in contrast to the feeling they experience with some healthcare professionals, where they sometimes feel judged:

"When she has diabetes, and I have diabetes, we are kind of conspirators (...) Then the relationship becomes a little closer compared to if it was, for example, a doctor who probably always is set on keeping a distance (...) And I like that. That you are not judged all the time." (Peer, outcomes achieved)

ICAMO-models

Based on our empirical findings, we revised our initial ICAMO model. We developed two ICAMO models (Figs. 2 and 3), to illustrate how the identified contextual factors in peers' everyday lives either facilitated or hindered their interactions in the intervention, thus affecting how the mechanisms within the peers were at stake. Figure 2 illustrates the ICAMO for the peers who achieved the intended outcomes and Fig. 3 illustrates the ICAMO for the peers who did not achieve these outcomes. 


\section{Discussion}

\section{Discussion of the findings}

In this multi-method case study, we contribute with novel findings on the mechanisms that generated outcomes in a Danish peer support intervention for socially vulnerable people with T2D (peers). Furthermore, we provide in-depth insights into how individual contextual factors in peers' everyday lives affected how the mechanisms were at stake. By categorising the peers depending on whether these contextual factors facilitated or hindered the mechanisms to generate outcomes, we support Pawson \& Tilley's (26) notion that mechanisms only operate when the circumstances are right and that effective interventions depend on contextual awareness.

The study population in this case study represents the target group we would like to examine, as their sociodemographic characteristics are consistent with the results from the initial quantitative analysis conducted within the CCDC to define socially vulnerable people with T2D (22).

Compared to our initial ICAMO model, our analysis pinpoints two groups of mechanisms that improved the peers' DSM and use of healthcare services ('perceived needs and readiness' and 'encouragement and energy'). Independent of the influence from the contextual factors, a third mechanism, 'experience of social and emotional support', was identified within all peers to increase self-care awareness (output).

Although we present the mechanisms separately, we consider them interrelated. For example, we found that the peer supporters' encouragement combined with the peers' levels of energy only led to health behaviour changes in DSM if the peers had a perceived need and sufficient readiness to adopt and maintain healthier eating and exercise patterns. Furthermore, we found that the implementation of a minimum of two of the three intervention activities was needed to activate the outcome-generating mechanisms. As described by Pawson \& Tilley, it is often impossible to find a key mechanism that is overarching and enables the outcome. Thus, the interaction of the intervention components, mechanisms and context as a whole facilitated the outcomes (26). Furthermore, we found four contextual factors in peers' everyday lives that either facilitated or hindered the mechanisms: 'occupation and financial situation', 'health condition', 'energy' and 'other life events'. Not all contextual factors were present in all cases. As argued by Dalkin et al. (39) and Craig et al. (24), the distinction between context and mechanism can be difficult to make. We experienced this challenge, especially as both our mechanism and context focus were on the peers. For example, 'energy' arose both as a mechanism and contextual factor, as it was a product of a range of different individual contextual factors.

Relating our results to existing literature on peer support for people with T2D is challenging. Many peer support interventions contain other approaches (e.g., group- or telephone-based) targeted to other populations (not socially vulnerable). Moreover, they are delivered by other types of stakeholders (e.g., clinics, churches and community organisations) and providers (peer educators, community health workers and peer-partners) $(9,13,46)$. Furthermore, they are implemented in other national contexts with other welfare systems and thus differ markedly from the Danish context. For example, in the US, most 
peer support interventions are community-based and contain diabetes education (47). Thus, they provide diabetes rehabilitation services similar to those that Danish municipalities are responsible for (31). Finally, the existing studies on peer support have either focused on measuring the intervention effect (effect evaluation)(13) or fidelity issues in process evaluations (48). Thus, our focus on the outcomegenerating mechanisms and contextual factors influencing this process is novel to this field. However, there seem to be some recurrent characteristics that might be general for the implementation of peer support interventions. A scoping review on community-based peer-led health promotion programmes (not specific to T2D) reported similar findings to this study. For example, peers' health conditions, work situations and beliefs towards the programme according to their needs were found as contextual factors to programme participation and engagement (49). These contextual factors are also reported in the existing literature on $\operatorname{DSM}(15,16,50)$. Likewise, lack of energy $(16,51)$ and the importance of social and emotional support have been addressed $(51,52)$.

Strengths and limitations of the study

This study has several strengths. First, it builds on a realist evaluation approach to identify the mechanisms that generate the intended outcomes. Using the ICAMO framework (27) to structure our analytical findings, we demonstrate how each intervention activity activates different mechanisms within the peers. Furthermore, we analyse how they, in interaction with the specific context, generate different cognitive (outputs) and behavioural changes (outcomes). These are findings that would otherwise not be possible to discover through more classic RCT-studies. Second, the use of case studies, which was recommended in the RE approach when analysing complex interventions $(45,53)$, allowed us to test our initial ICAMO model and verify whether propositions in the ICAMO could be reproduced in different contexts. Third, it triangulates different data collection sources and both qualitative and quantitative methods, enhancing the credibility of the findings. Further, with our relatively large sample size of nine cases with four different types of informants in each $(n=25)$, we achieved sufficient information power to test and revise our ICAMO models (54). However, the study has some limitations. First, due to the COVID19 pandemic, seven interviews (three with peers) were conducted via telephone. Comparing these with the other interviews, we do not consider that this had consequences for the analysis. Second, we only investigated contextual factors in peers' everyday lives. Another context focus (e.g., organisational or interpersonal contextual factors) (38) had provided us with other analytical findings. Third, we only used the ICAMO framework to structure our findings. Thus, we did not apply the realist interview. However, we did not consider this approach relevant, as we had in-depth knowledge from all key stakeholders to understand their perspectives. Further, using the ICAMO framework contributes to the understanding of the interaction between context and mechanisms, and it may be relevant for other researchers to apply this when studying interventions targeting socially vulnerable groups. We believe that the in-depth data and analysis were valuable in generating contextually relevant evidence for improving the efficiency and effectiveness of peer support targeting socially vulnerable people with T2D and informing policy decisions for this group.

Implication for practice and future research 
The role of context in population health intervention research is increasingly acknowledged. In a guidance by Craig et. al., the context can be taken into account by ensuring that the intervention is adaptable to the target group, for example, by conducting a needs assessment and testing the intervention in different contexts to uncover contextual factors that could affect the outcomes (24). Therefore, we contribute moving the field forward beyond having contextual awareness of the target population $(23,24)$. The focus on the target group in the present study revealed that even though the Together on Diabetes intervention was developed to support socially vulnerable people with T2D, the most vulnerable people did not achieve the intended outcomes. Although, this group did not improve their DSM and/or increase their use of healthcare services, the peer supporters' social and emotional support was still experienced as valuable and increased self-care awareness. We, therefore, find the social and emotional support important but not sufficient when it comes to a change in DSM and the use of healthcare services. For this specific vulnerable group, professional support coordinated between the health sector and social services is needed due to the individual contextual factors that characterise this target group.

\section{Conclusion}

In this study, we explored the mechanisms generating outcomes in a Danish peer support intervention targeted to improve diabetes self-management and the use of healthcare services among socially vulnerable people with type 2-diabetes (peers). Using a realist evaluation approach, our study contributes novel, in-depth findings to a research field that needs more knowledge on how to reach socially vulnerable people with T2D with complex health interventions; people that the healthcare system, even in a universal welfare system such as in Denmark, does not reach. We found that the peer support intervention only activated mechanisms generating the intended outcomes if contextual factors in peers' everyday lives facilitated their engagement in the intervention. Thus, we highlight the importance of having contextual awareness on the target group in designing and evaluating peer support interventions for socially vulnerable people with type 2-diabetes.

\section{Abbreviations}

DSM

diabetes self-management

$\mathrm{T} 2 \mathrm{D}$

type 2-diabetes

\section{Declarations}

\section{Ethics approval and consent to participate}

The study was approved by the Danish Data Protection Agency (Rec. No: 2015-55-0630) and followed the codes of ethics in the Helsinki II Declaration. An ethical application was sent to The Research Ethics Committee for SCIENCE and HEALTH, at the Capital Region of Denmark. The Research Ethics Committee 
reported that they did not identify any ethical hindrances in conducting the study (Id. No: 18029206) and decided that no formal ethical approval was needed.

All participants received written and verbal information about the study and gave written consent to participate. They were guaranteed anonymity and informed that they could withdraw from the study at any time, should they wish to do so.

\section{Consent for publication}

Not applicable

\section{Availability of data and material}

The data/transcripts used during this study are available from the corresponding author upon reasonable request.

\section{Competing interests}

The authors declare that they have no competing interests

\section{Funding}

This study was jointly funded by Cities Changing Diabetes, Novo Nordisk A/S (25\%), the Center for Diabetes (25\%) and the Innovations Fund Denmark (50\%) (Application number: 7091-00008B). The sponsors had no role in the design, execution, interpretation, or writing of the study, or in the decision to publish the results.

\section{Authors' contributions}

SG, UC and CG designed the study. SG and GM collected all data material, SG drafted the manuscript, and all the authors revised the manuscript for intellectual content. SV and UC contributed to the analysis and interpretation of data. GM, CG, UC and SV contributed with critical reviews of the work. All authors read, commented on and approved the final manuscript.

\section{Acknowledgements}

The authors would like to thank the project manager, diabetes nurse, peers and peer supporters who participated in the study. Furthermore, the authors would like to thank the project manager for the 
recruitment of peers and peer supporters. Likewise, we would like to thank the diabetes nurse for collecting the survey data among the peers. We also thank Majken P. Pedersen for contributions to the conducting of interviews with the peer supporters and Louise Hesseldal for valuable comments on an earlier version of this paper.

\section{References}

1. Caro JF, Fisher EB. A solution might be within people with diabetes themselves. Fam Pract. 2010;27 Suppl 1(September 2008):1-2.

2. Johansson T, Keller $\mathrm{S}$, Winkler $\mathrm{H}$. Can a peer support intervention improve type 2 diabetes outcomes ? Diabetes Voice. 2014;59(June):2-5.

3. Werfalli M, Werfalli M, Raubenheimer PJ, Engel M, Musekiwa A, Bobrow K, et al. The effectiveness of peer and community health worker-led self-management support programs for improving diabetes health-related outcomes in adults in low- and-middle-income countries: A systematic review. Syst Rev. 2020;9(1).

4. Brownson CA, Heisler M. The role of peer support in diabetes care and self-management. Patient. 2009;2(1):5-17.

5. WHO. Peer Support Programmes in Diabetes. 2007;43.

6. Heisler M. Building Peer Support Programs to Manage Chronic Disease: Seven Models for Success. 2006. 1-42 p.

7. Fisher EB, Ballesteros J, Bhushan N, Coufal MM, Kowitt SD, Manuela McDonough A, et al. Analysis \& commentary: Key features of peer support in chronic disease prevention and management. Health Aff. 2015;34(9):1523-30.

8. Deng K, Ren Y, Luo Z, Du K, Zhang X, Zhang Q. Peer support training improved the glycemic control, insulin management, and diabetic behaviors of patients with type 2 diabetes in rural communities of Central China: A randomized controlled trial. Med Sci Monit. 2016;22:267-75.

9. Heisler M. Overview of peer support models to improve diabetes self-management and clinical outcomes. Diabetes Spectr. 2007;20(4):214-21.

10. Lorig K, Ritter PL, Villa FJ, Armas J. Community-based peer-led diabetes self-management: A randomized trial. Diabetes Educ. 2009;35(4):641-51.

11. Fisher EB, Boothroyd RI, Elstad EA, Hays L, Henes A, Maslow GR, et al. Peer support of complex health behaviors in prevention and disease management with special reference to diabetes: systematic reviews. Clin diabetes Endocrinol [Internet]. 2017;3:4. Available from: http://www.ncbi.nlm.nih.gov/pubmed/28702258.

12. Paul GM, Smith SM, Whitford DL, O'Shea E, O'Kelly F, O'Dowd T. Peer support in type 2 diabetes: A randomised controlled trial in primary care with parallel economic and qualitative analyses: Pilot study and protocol. BMC Fam Pract. 2007. 
13. Zhang X, Yang S, Sun K, Fisher EB, Sun X. How to achieve better effect of peer support among adults with type 2 diabetes: A meta-analysis of randomized clinical trials. Patient Educ Couns [Internet]. 2016;99(2):186-97. Available from: http://dx.doi.org/10.1016/j.pec.2015.09.006.

14. Sokol R, Fisher E. Peer Support for the Hardly Reached: A Systematic Review. Am J Public Health. 2016;106(7):1308.

15. Christensen NI, Drejer S, Burns K, Lundstrøm SL, Hempler NF. A qualitative exploration of facilitators and barriers for diabetes self-management behaviors among persons with type 2 diabetes from a socially disadvantaged area. Patient Prefer Adherence. 2020;14:569-80.

16. Christensen U, Kristensen EC, Malling Hvid GM. Vulnerability Assessment in Copenhagen, Cities Changing Diabetes. Copenhagen; 2016.

17. Agardh E, Allebeck P, Hallqvist J, Moradi T, Sidorchuk A. Type 2 diabetes incidence and socioeconomic position: A systematic review and meta-analysis. Int J Epidemiol. 2011;40(3):804-18.

18. Espelt A, Arriola L, Borrell C, Larranaga I, Sandin M, Escolar-Pujolar A. Socioeconomic Position and Type 2 Diabetes Mellitus in Europe 1999-2009: a Panorama of Inequalities. Curr Diabetes Rev. 2011.

19. DIKE. Social ulighed, sundhed og sygdom. 2020.

20. Sorts $\varnothing$ C, Lauridsen J, Emneus M, Green A, Jensen PB. Socioeconomic inequality of diabetes patients' health care utilization in Denmark. Health Econ Rev. 2017.

21. Walker RJ, Gebregziabher M, Martin-Harris B, Egede LE. Independent effects of socioeconomic and psychological social determinants of health on self-care and outcomes in Type 2 diabetes. Gen Hosp Psychiatry. 2014;36(6):662-8.

22. Holm A, Ledgaard GS, Andersen ME, Jørgensen, and Finn Diderichsen. Is the Rule of Halves Framework Relevant for Diabetes Care in Copenhagen Today? A Register-Based Cross-Sectional Study. BMJ Open. 2018;8(11):1-10.

23. Moore GF, Audrey S, Barker M, Bond L, Bonell C, Hardeman W, et al. Process evaluation of complex interventions: Medical Research Council guidance. BMJ. 2015;350.

24. Craig P, Ruggiero E, Di, Frohlich KL, Mykhalovskiy E, White M, Campbell R, et al. Taking account of context in population health intervention research: guidance for producers, users and funders of research. 2018; Available from: https://doi.org/10.3310/CIHR-NIHR-01.

25. Pinnock H, Barwick M, Carpenter CR, Eldridge S, Grandes G, Griffiths CJ, et al. Standards for Reporting Implementation Studies (StaRI) Statement. BMJ [Internet]. 2017;356(March):1-9. Available from: http://dx.doi.org/doi:10.1136/bmj.i6795.

26. Pawson R, Tilley N Realistic Evaluation. SAGE Publ. 1997.

27. Mukumbang FC, Marchal B, Van Belle S, van Wyk B. Using the realist interview approach to maintain theoretical awareness in realist studies. Qual Res. 2019.

28. Jonsson F, Goicolea I. "We believe in you, like really believe in you": Initiating a realist study of (re)engagement initiatives for youth not in employment, education or training with experiences from 
northern Sweden. Eval Program Plann [Internet]. 2020;83(April):101851. Available from: https://doi.org/10.1016/j.evalprogplan.2020.101851.

29. Mukumbang FC, Marchal B, Van Belle S, Van Wyk B. Unearthing how, why, for whom and under what health system conditions the antiretroviral treatment adherence club intervention in South Africa works: A realist theory refining approach. BMC Health Serv Res. 2018;18(1):1-15.

30. Olejaz M, Juul Nielsen A, Rudkjøbing A, Okkels Birk H, Krasnik A, Hernández-Quevedo C. Denmark health system review. Health Syst Transit. 2012;14(2).

31. Snorgaard O, Jensen J, Laursen M. et. al. Forløbsprogram TYPE 2 Diabetes. 2009;112.

32. Health. and Care Adminstarion. Action Plan for Type 2-Diabetes. 2019.

33. David Napier A, Nolan JJ, Bagger M, Hesseldal L, Volkmann AM. Study protocol for the Cities Changing Diabetes programme: A global mixed-methods approach. BMJ Open. 2017;7(11):1-7.

34. Cities Changing Diabetes. ACTION ON How cities are building a healthier future. 2020.

35. Fisher BE, Boothroyd IR, Coufal MM, Baumann CL, Mbanya JC, Rotheram-Borus MJ, et al. Peer support for self-management of diabetes improved outcomes in international settings. Vol. 31, Health Affairs. 2012. p. 130-9.

36. Fisher EB, Ballesteros J, Bhushan N, Coufal MM, Kowitt SD, Manuela McDonough A, et al. Analysis \& commentary: Key features of peer support in chronic disease prevention and management. Health Aff. 2015;34(9):1523-30.

37. Kræftens B. Navigatorprogram for kræftpatienter. 2015.

38. Pollitt C. Ray, Pawson. (2013) The Science of Evaluation: A Realist Manifesto. Int Rev Adm Sci. 2013.

39. Dalkin SM, Greenhalgh J, Jones D, Cunningham B, Lhussier M. What's in a mechanism? Development of a key concept in realist evaluation. Implement Sci. 2015;10(1):1-7.

40. Yin RK. Applications of case study research. Appl Soc Res Methods Ser. 2013.

41. Stake R. Multiple Case study analysis. Computational Methods and Function Theory. 2006.

42. Christensen Al, Ekholm O, Juel K, Glümer C, Andreasen AH, Hvidberg MF, et al. The Danish National Health Survey 2010. Study design and respondent characteristics. Scand J Public Health. 2012.

43. Castleberry A. NVivo qualitative data analysis Software; QSR International Pty Ltd. Version 10, 2012. Am J Pharm Educ. 2014.

44. Malterud K. Systematic text condensation: A strategy for qualitative analysis. Scand J Public Health. 2012.

45. Wong G, Westhorp G, Manzano A, Greenhalgh J, Jagosh J, Greenhalgh T. RAMESES II reporting standards for realist evaluations. BMC Med [Internet]. 2016;14(1):1-18. Available from: http://dx.doi.org/10.1186/s12916-016-0643-1.

46. Aziz Z, Riddell MA, Absetz P, Brand M, Oldenburg B, Dunbar JA, et al. Peer support to improve diabetes care: An implementation evaluation of the Australasian Peers for Progress Diabetes Program. BMC Public Health. 2018;18(1):1-12. 
47. American Diabetes Association [Internet]. [cited 2021 Jan 5]. Available from: https://www.professional.diabetes.org/content-page/diabetes-support-directory.

48. Saunders RP, Evans MH, Joshi P. Developing a Process-Evaluation Plan for Assessing Health Promotion Program Implementation: A How-To Guide. Health Promot Pract. 2005.

49. Lorthios-Guilledroit A, Richard L, Filiatrault J. Factors associated with the implementation of community-based peer-led health promotion programs: A scoping review. Eval Program Plann [Internet]. 2018;68(April 2017):19-33. Available from: https://doi.org/10.1016/j.evalprogplan.2018.01.008.

50. Nam S, Chesla C, Stotts NA, Kroon L, Janson SL. Barriers to diabetes management: Patient and provider factors. Diabetes Res Clin Pract. 2011;93(1):1-9.

51. Wilkinson A, Whitehead L, Ritchie L. Factors influencing the ability to self-manage diabetes for adults living with type 1 or 2 diabetes. Int J Nurs Stud [Internet]. 2014;51(1):111-22. Available from: http://dx.doi.org/10.1016/j.ijnurstu.2013.01.006.

52. Goetz K, Szecsenyi J, Campbell S, Rosemann T, Rueter G, Raum E, et al. The importance of social support for people with type 2 diabetes - A qualitative study with general practitioners, practice nurses and patients. Psychosoc Med. 2012;9:Doc02-2.

53. Peter Craig P, Dieppe S, Macintyre S, Michie I, Nazareth M, Petticrew. Developing and evaluating complex interventions: Following considerable development in the field since 2006, MRC and NIHR have jointly commissioned an update of this guidance to be published in 2019. 2006; Available from:

54. Malterud K, Siersma VD, Guassora AD. Sample Size in Qualitative Interview Studies: Guided by Information Power. Qual Health Res. 2016.

\section{Figures}




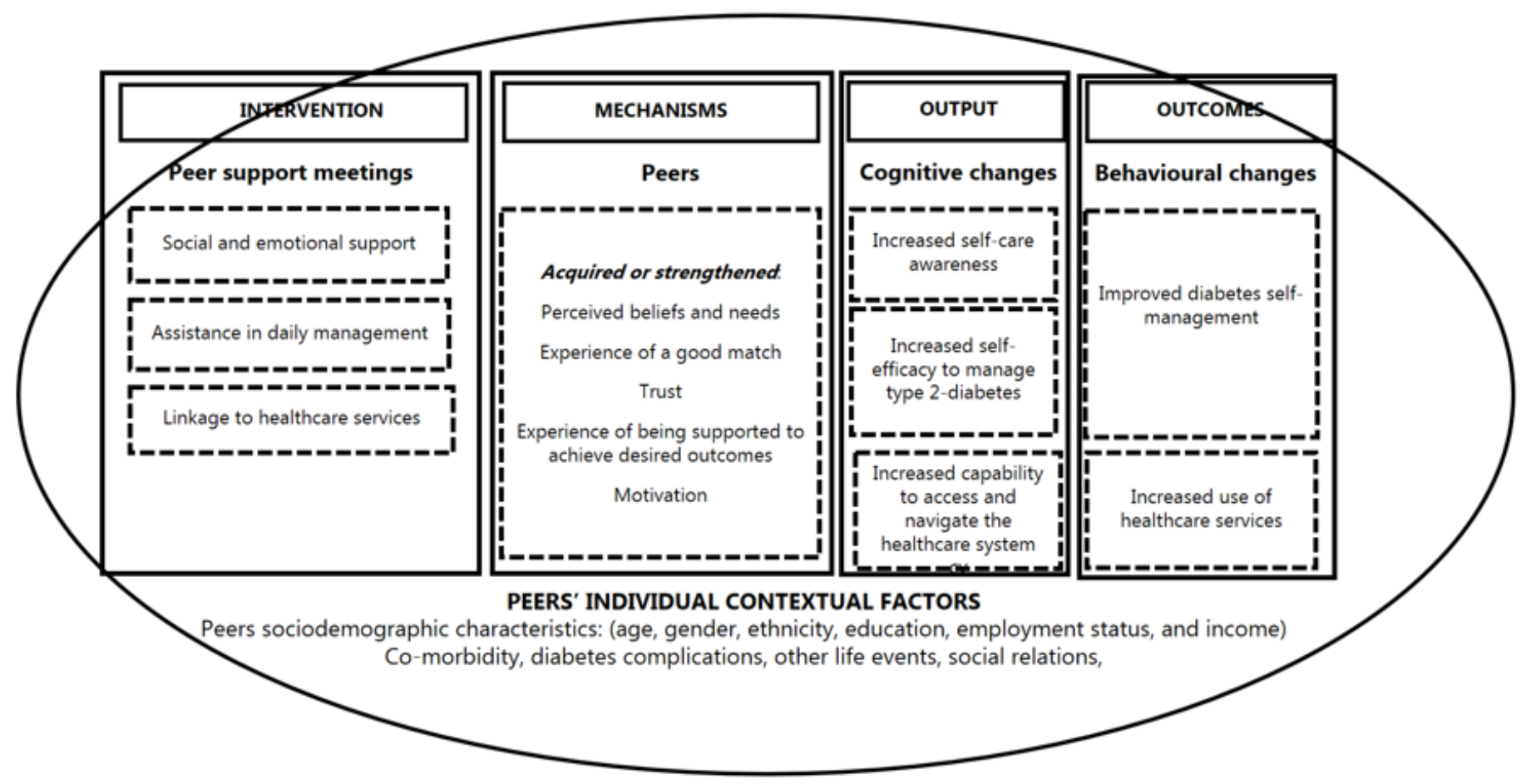

Figure 1

Initial ICAMO model

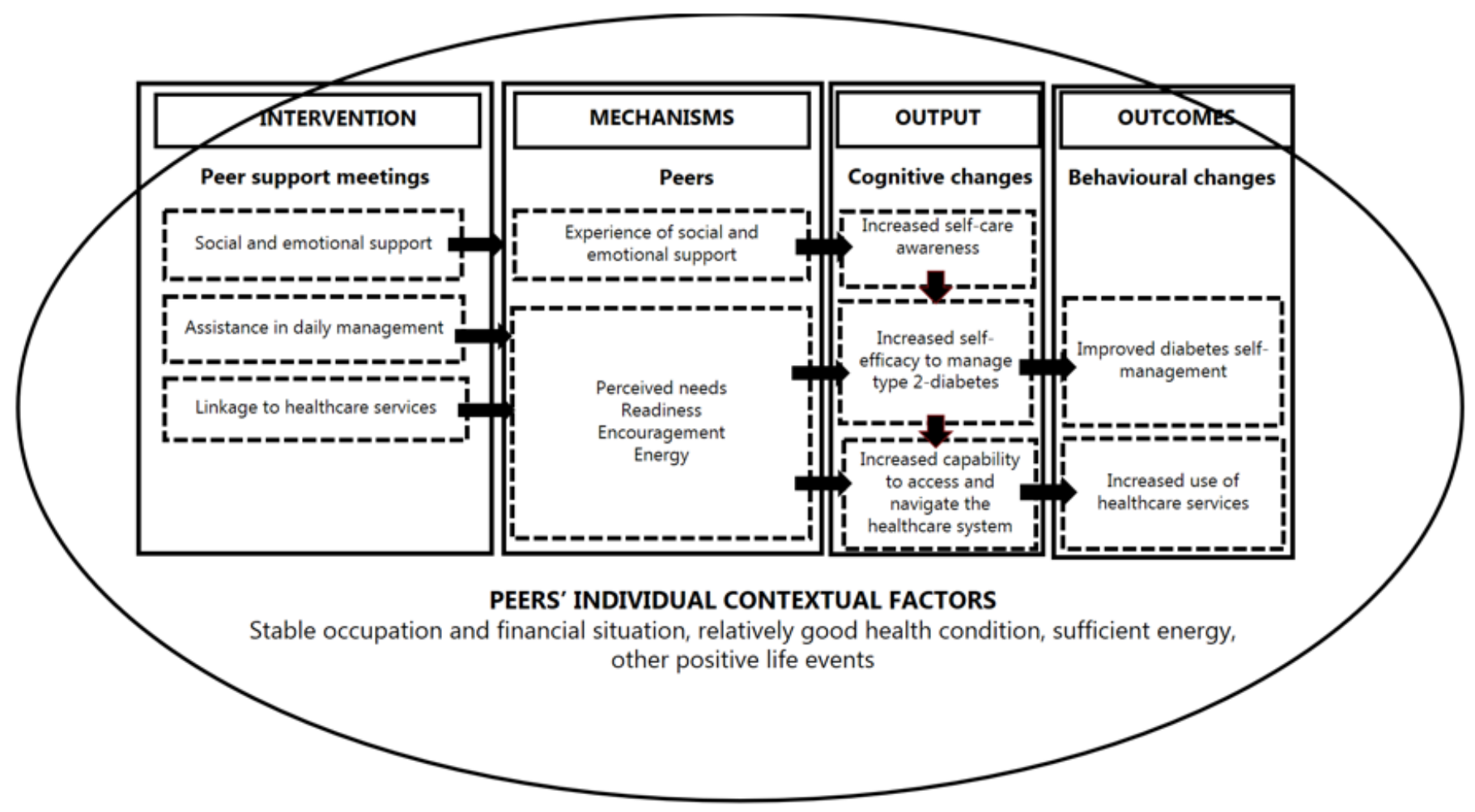

Figure 2

ICAMO model for peers who achieved the intended outcomes 


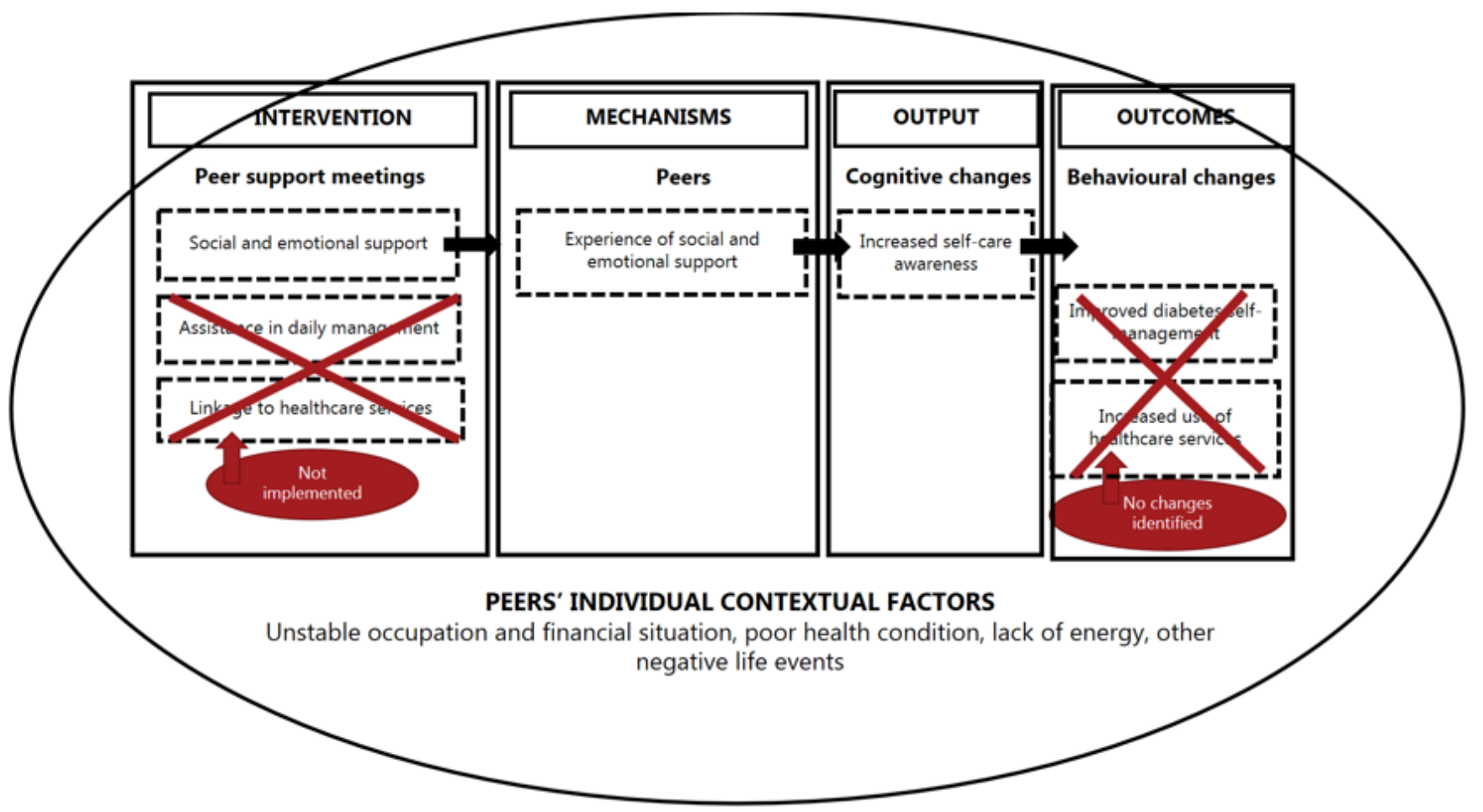

Figure 3

ICAMO model for peers who did not achieve the intended outcomes

\section{Supplementary Files}

This is a list of supplementary files associated with this preprint. Click to download.

- StaRIchecklist.docx 